\title{
Adiabatic transformation of continuous waves into trains of pulses
}

\author{
J. M. Soto-Crespo, ${ }^{1}$ N. Devine, ${ }^{2}$ and N. Akhmediev ${ }^{2}$ \\ ${ }^{1}$ Instituto de Óptica, C.S.I.C., Serrano 121, 28006 Madrid, Spain \\ ${ }^{2}$ Optical Sciences Group, Research School of Physical Sciences and Engineering, \\ The Australian National University, Canberra ACT 0200, Australia
}

(Received 2 November 2016; published 10 August 2017)

\begin{abstract}
Periodic structures may grow in both conservative and dissipative systems. A multiplicity of examples can be found in nature and in the laboratory. However, periodic structures may grow and decay. We show that the effects of dissipation are essential for these structures to remain. Using the nonlinear Schrödinger equation and its extensions as basic examples of conservative and dissipative systems we show that there are two ways of adiabatic transformations of a continuous-wave solution into a train of pulses.
\end{abstract}

DOI: 10.1103/PhysRevA.96.023825

\section{INTRODUCTION}

Periodic structures grow spontaneously in various physical contexts, such as gravel road corrugation [1,2], formation of convective cells [3], pattern formation in optical cavities [4], and in bulk optical media [5]. For these systems, there are two important questions to answer: When can we expect a stable periodic pattern to appear from a uniform initial state? Can we control this process? The physics behind each of the listed phenomena differs. However, there must be common mechanisms that are responsible for the transition from a homogeneous state or a plane wave to a periodic pattern. Finding them is the challenge that we address in this paper.

Normally, it is a modulational instability (MI) which starts the growth of periodic structures from uniformly constant initial conditions [6,7]. The initial stage of this growth is simply exponential. However, what happens beyond the exponential growth is a complicated process [8-10]. In some cases, these structures may disappear the same way as they grew. Such return process is related to the Fermi-Pasta-Ulam recurrence [11-13]. This mostly happens in conservative and integrable systems. In other cases, once the structure appears, it remains indefinitely. Examples include periodic ripples in sand dunes [14] and deserts [15] and skin pigment patterns in biology [16]. The emergence of stable periodic structures is common for dissipative systems.

These general principles are well used in modern technology. Examples of practical devices based on this transformation are passively mode-locked lasers $[17,18]$ where a continuous pump is transformed into a train of pulses and microcavities for generation of frequency combs [19-23]. The latter have important applications in time and frequency metrology $[24,25]$. The large variety of these devices requires an individual modeling. However, there is a master equation approach that may unify their mathematical description. The role of the master equation is played by the complex GinzburgLandau equation with parameters averaged across the cavity period $[17,18]$. As we show here, the transition from the conservative system represented by the nonlinear Schrödinger equation (NLSE) to the dissipative system represented by the complex Ginzburg-Landau equation (CGLE) is the key for understanding the process of transformation. As the CGLE has a wide range of applicability in physics [26], the concept presented here can be extended to many other physical situations.
We start with the NLSE which is used in many physical contexts and can be considered as one of the fundamental equations of nonlinear wave physics. In dimensionless form, it reads

$$
i \psi_{\xi}+\frac{1}{2} \psi_{\tau \tau}+|\psi|^{2} \psi=0,
$$

where $\psi$ is the function describing the wave envelope of the field of interest, $\xi$ is the evolution variable (time or distance along propagation), and $\tau$ is the transverse variable (retarded time in the case of optical fibers). The best known solution of the NLSE is the soliton that has been observed on water surface [27], in optical fibers [28], and in many other physical situations. Its main feature is its robustness [29]. Solitons propagate large distances, withstanding dispersion that usually destroys pulses in linear systems. Solitons do exist in many other systems where nonlinearity and dispersion are balanced for a pulselike solution [30-32]. Consequently, the notion of soliton is relevant to a much wider class of systems [33-35] than was initially thought.

In addition to solitons, the NLSE has a number of periodic solutions that are no less important in physics. In the simplest case, these solutions are periodic in the $\tau$ variable. They can also be periodic in evolution, i.e., along the $\xi$ variable. In the latter case, we dub them as "doubly periodic" solutions. In doing this, we ignore the periodicity related to the carrier wave $\exp \left(i q_{o}^{2} \xi\right)$, i.e., we consider only the periodicity of the envelope function. Below, we give the analytical expressions for two of these solutions as this is important for understanding the results of our numerical simulations.

\section{EXACT PERIODIC NLSE SOLUTIONS}

One of the doubly periodic solutions of the NLSE (denoted as - type A) has the following form [36]:

$$
\psi=q_{0} \frac{k \operatorname{sn}(\zeta, k)-i C(\tau, k) \operatorname{dn}(\zeta, k)}{k[1-C(\tau, k) \operatorname{cn}(\zeta, k)]} e^{\left(i q_{0}^{2} \xi\right)},
$$

where cn, sn, and dn are Jacobi elliptic functions [37], $k$ being their modulus $(0<k<1), \zeta=\frac{q_{0}^{2} \xi}{k}$,

$$
C(\tau, k)=\sqrt{\frac{k}{1+k}} \operatorname{cn}\left(\frac{\sqrt{2} q_{0}}{\sqrt{k}} \tau, \sqrt{\frac{1-k}{2}}\right),
$$




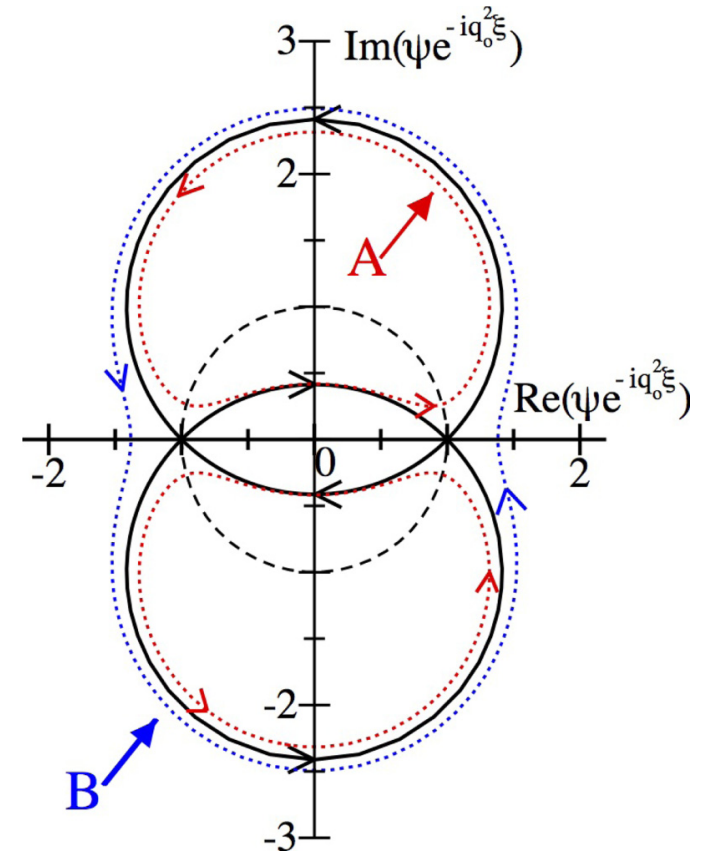

FIG. 1. Phase portrait of the dynamics near the heteroclinic orbits (black bold circles) for doubly periodic NLSE solutions (2) and (5) (outer blue and inner red dotted curves).

and $q_{0}$ is a real scaling parameter. The modulus of the elliptic function $\mathrm{cn}$ in (3) is $\sqrt{\frac{1-k}{2}}$.

Each solution of this family is periodic in both $\tau$ and $\xi$ with periods that can be adjusted to the initial conditions and the numerical results by varying $k$ and $q_{0}$. The $\xi$ evolution of this solution can be conveniently represented on the complex plane of $\psi(\xi, \tau)$. For a fixed $\tau=0$, the trajectory of the curve $\psi(\xi, 0) e^{-i q_{0}^{2} \xi}$ is illustrated in Fig. 1 by the red curve and denoted by the arrow having the "A" label. The exponential factor $e^{-i q_{0}^{2} \xi}$ eliminates the fast rotation of the curve around the origin. The trajectory shows that the solution is indeed periodic. Taking the parameter $k$ to the limit $k \rightarrow 1$ transforms this solution into a heteroclinic orbit shown by the black circle. Then the trajectory gets closer to the two saddle points \pm 1 , and the period in $\xi$ tends to infinity. The similar trajectory in the lower half-plane is calculated for $\tau=T / 2$.

In the limit $k=1$, elliptic Jacobi functions are transformed into either trigonometric or hyperbolic functions and Eq. (2) reduces to the solution which is known as Akhmediev breather (AB) [38-41],

$$
\psi=\sqrt{2} q_{0} \frac{-\sqrt{2} \sinh \left(q_{0}^{2} \xi\right)+i \cos \left(\sqrt{2} q_{0} \tau\right)}{2 \cosh \left(q_{0}^{2} \xi\right)-\sqrt{2} \cos \left(\sqrt{2} q_{0} \tau\right)} e^{i q_{0}^{2} \xi}
$$

It has an infinite period in $\xi$ and is the above-mentioned heteroclinic orbit. This solution starts from the continuouswave $(\mathrm{cw})$ solution $\psi=q_{0} e^{i q_{0}^{2} \xi}$ of the NLSE at $\xi=-\infty$. At finite negative $\xi$, it gains a periodic modulation which grows exponentially. This part of the evolution is known as modulation instability. The growth reaches its zenith at $\xi=0$, and then returns back to the $\mathrm{cw}$ solution $\psi=-q_{0} e^{i q_{0}^{2} \xi}$ at $\xi=\infty$ acquiring a phase shift $\pi$. This return behavior is the Fermi-Pasta-Ulam recurrence for the NLSE [11-13]. Indeed, in the spectral domain, at the beginning $\xi=-\infty$ and at the end of evolution $\xi=+\infty$, all the energy is concentrated in a $\mathrm{cw}$ which is a single normal mode of the system while at $\xi=0$, the energy is spread between all components of the equidistant triangular comb spectrum [39].

The AB solution (4) serves as a separatrix between two types of doubly periodic solutions. The periodic solution, that we shall denote as B type, located on the other side of the separatrix (4) has the form [36,39]

$$
\psi=q_{0} \kappa \frac{A(\tau) \operatorname{cn}\left(q_{0}^{2} \xi, \kappa\right)+i \sqrt{1+\kappa} \operatorname{sn}\left(q_{0}^{2} \xi, \kappa\right)}{\sqrt{1+\kappa}-A(\tau) \operatorname{dn}\left(q_{0}^{2} \xi, \kappa\right)} e^{\left(i q_{0}^{2} \xi\right)},
$$

where

$$
A(\tau)=\mathrm{cd}\left[\sqrt{(1+\kappa)} q_{0} \tau, \sqrt{\frac{(1-\kappa)}{(1+\kappa)}}\right],
$$

and elliptic Jacobi function $c d=c n / d n$. The modulus of elliptic functions in Eq. (5) is $\kappa(0<\kappa<1)$ while in Eq. (6), it is $\sqrt{\frac{(1-\kappa)}{(1+\kappa)}}$. When $\kappa \rightarrow 1$, solution (5) reduces to (4).

The trajectory for the solution (5) is shown in Fig. 1 by the blue dotted curve labeled B. As the solutions (2) and (5) are located on different sides of the separatrix (4), they are qualitatively different. Trajectory A rotates on one side of the complex plane and does not add the rotation to the phase of the solution while the trajectory B rotates around the origin thus gaining a phase difference of $2 \pi$ on each period of oscillation. Wave profiles of these two solutions are shown in Fig. 2. The solution (5) keeps maxima of the periodic function along the $\tau$ axis at the same position while the solution (2) has its maxima
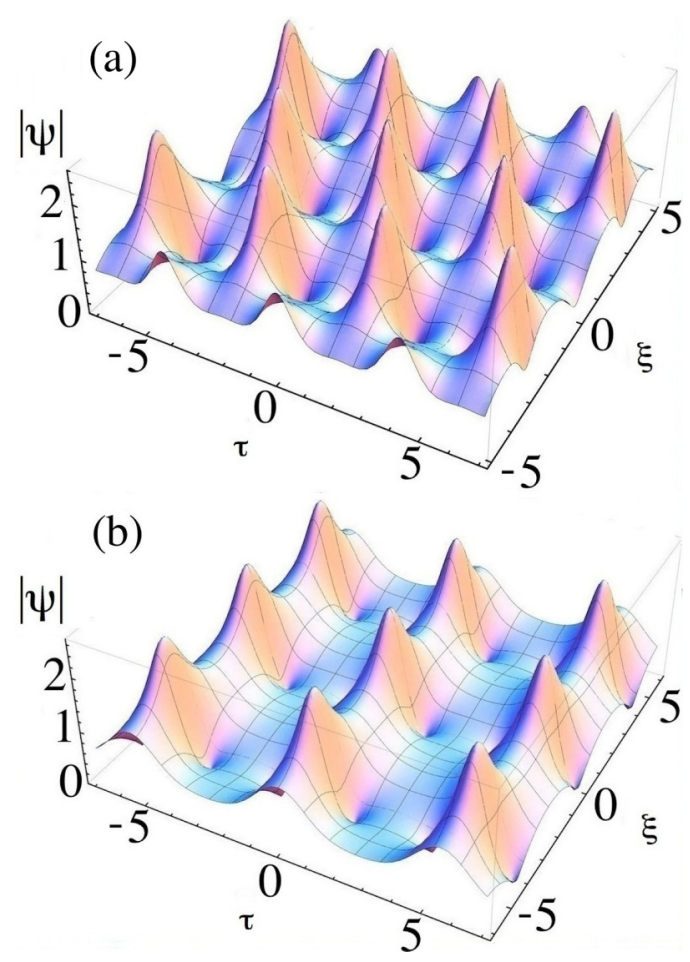

FIG. 2. (a) A-type (2) and (b) B-type (5) doubly periodic solutions on the $(\tau, \xi)$ plane. In each case, modulus $(k$ or $\kappa)=0.7$ and $q_{0}=1$. 
located alternatively between two positions. This can be seen in Figs. 2(a) and 2(b).

In addition to the doubly periodic solutions listed above, there are two families of stationary periodic solutions of the NLSE. The family of type 1 solutions is

$$
\psi=q_{0}(1+\kappa) \operatorname{dn}\left(q_{0}(1+\kappa) \tau, \frac{2 \sqrt{\kappa}}{1+\kappa}\right) e^{\left[i q_{0}^{2}\left(1+\kappa^{2}\right) \xi\right]}
$$

while the family of type 2 solutions is given by

$$
\psi=\frac{\kappa q_{0}}{\sqrt{\kappa^{2}-1 / 2}} \operatorname{cn}\left(\frac{q_{0} \tau}{\sqrt{\kappa^{2}-1 / 2}}, \kappa\right) e^{\left(i q_{0}^{2} \xi\right)} .
$$

The dn elliptic Jacobi function does not change the sign in $\tau$ and the solution (7) has no zeros. In contrast, the cn function does change the sign along $\tau$. Therefore, the solution (8) does have zeros in the transverse direction. The plots of these two functions are shown in Fig. 7 by red dotted curves. Importantly, solutions (7) and (8) can be represented as periodic trains of solitons [42].

\section{ROBUSTNESS OF PERIODIC ORBITS}

As the NLSE is integrable, its solutions are neutrally stable and normally are not transformed from one to another. Solutions are robust and small perturbations do not change them. In particular, soliton solutions are robust and their propagation is not hampered by the presence of perturbations [29]. Moreover, Mahnke and Mitschke [41] have shown that the Akhmediev breather solutions of the NLSE are also robust. They cannot be destroyed by small perturbations of the solution. These solutions can only be changed if the governing equation, i.e., the NLSE, is perturbed. The studies of Calini and Schober [43] provided another confirmation of this result. Namely, they showed that the ABs and related solutions of the NLSE are stable within the frame of the NLSE.

Our own numerical simulations, once again, confirmed these results. Moreover, in our simulations, we extended the above conclusion to the doubly periodic solutions of the NLSE given by Eqs. (2) and (5). These solutions cannot be modified by adding small perturbations. On the other hand, small perturbations of the governing equation may significantly distort the solutions. The perturbations that we use are all dissipative. Thus, even if they are small, they introduce a qualitative change to the system. On the other hand, the solutions of this dissipative system stay close to the solutions of the NLSE. The additional dissipative terms continuously transform a solution of the NLSE into another solution of the same equation. In other words, the solution evolves adiabatically from one NLSE solution to another.

What we have found is that the ABs and the nearby periodic solutions can be transformed into stable periodic trains. This means that a cw used as a pump, can be adiabatically transformed into a train of stationary solitons. Moreover, this transformation can be done in two ways, thus producing two different types of pulse trains. Nevertheless, simply linear gain or loss are not sufficient for such transformations. In order to show this, we started by adding a linear gain or loss perturbative term to the NLSE:

$$
i \psi_{\xi}+\frac{1}{2} \psi_{\tau \tau}+|\psi|^{2} \psi=i \delta \psi
$$

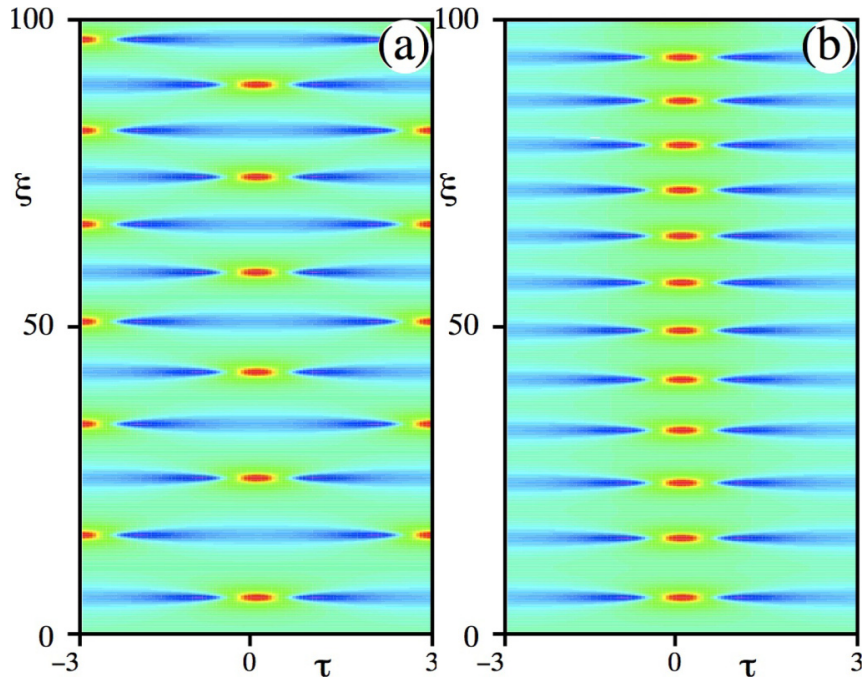

FIG. 3. False color plot of periodic solutions excited when $\delta$ is (a) negative and (b) positive. A-type and B-type solutions are excited in (a) and (b), respectively.

where $\delta$ accounts for linear losses (when $\delta$ is negative) or gain ( $\delta$ positive). We solved the above equation taking as initial condition a $\mathrm{cw}$ with a small periodic perturbation $\psi(\tau, 0)=1+0.01 \cos (2 \pi \tau / T)$, allowing modulation instability to develop. When $\delta=0$, the real finite perturbation 0.01 in this initial condition shifts the initial point from the saddle to the hyperbolic orbit of the B-type solution.

The presence of $\delta$, even being small, changes the dynamics. After leaving the saddle point, the periodic trajectories approach closely the $\mathrm{AB}$ orbit. The distance between the trajectories becomes so small that they can cross the separatrix and can be adiabatically transformed into the periodic solution of another type due to the loss or gain term added to the NLSE. Alternatively, the motion may slip from one periodic orbit to another of the same type. These changes depend on the sign of $\delta$.

Two examples are shown in Figs. 3(a) and 3(b). When $\delta$ is negative, $=-0.0001$, the evolution is shown in Fig. 3(a), while for the case with gain $(\delta=0.0001)$ it is shown in Fig. 3(b). Despite the smallness of $\delta$, the solution is transformed to the periodic one of A type in the first case and to the periodic solution of B type in the second case. On propagation up to $\xi=10000$, where the simulations have been stopped, no qualitative changes were observed. The motion remains periodic in $\xi$ with slightly changing period. This change shows that the trajectory slips continuously from one periodic orbit to another one.

An additional proof that the periodic A-type solution is excited when $\delta$ is negative is shown in Fig. 4. Here, we constructed the phase portraits of the solution on the complex plane. The three examples correspond to three different periods in $\tau$. The maximum growth rate of the instability $(\omega=\sqrt{2} / 2)$ corresponds to the period $T=4.4429$. The two other periods (4.6 and 4.2) correspond to neighboring frequencies. In all three cases, the trajectories remain close to the $\mathrm{AB}$ orbit and stay in the upper part of the complex plane which is the main feature of the A-type solution. 

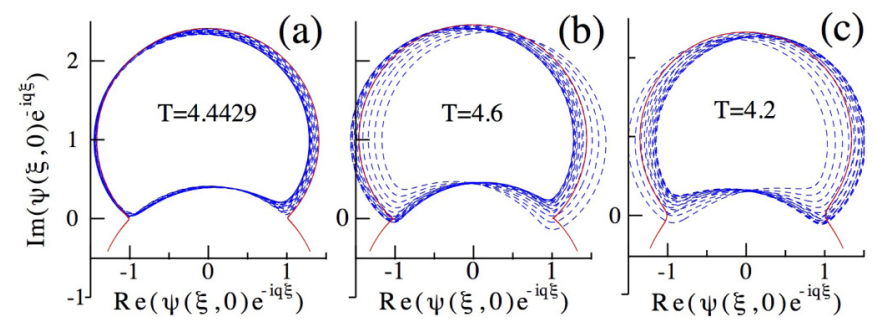

FIG. 4. Phase trajectories on the complex plane for the solutions of the NLSE with (dashed blue line, $\delta=-0.0001$ ) and without (red solid line) losses for three modulation periods $T$.

The solution slips from one periodic orbit to another with simultaneous shifts of the phase. Comparison with Fig. 2 shows once again that the three solutions stay within the A type in the interval $0<\xi<200$. In fact, they do so in a much larger $\xi$ interval, although the trajectories beyond this interval are not shown. Such transformations have been observed experimentally for waves in a water tank [44].

When alternating the linear term, the trajectories may cross the separatrix and slip to another type of solution. This happens when the parameter $\delta$ changes sign during the evolution. In order to show this, we used a periodic sinusoidal modulation of $\delta$ in $\xi$. If the periods of modulation and the solution are not commensurate, the changes may happen chaotically. Two examples of such dynamics are shown in Figs. 5(a) and 5(b). The two panels correspond to a periodic $\delta$ dependence on $\xi$ with different periods and amplitudes of modulation. Namely, in the case (a), we took $\delta=-0.02 \cos (0.2 \xi)$ while in the case (b), the dependence is $\delta=-0.09 \cos (0.8 \xi)$. In each case, the solution slipped from the $\mathrm{AB}$ trajectory to one of the periodic orbits and then jumped between the A-type and B-type orbits chaotically. The main conclusion out of these simulations is that for small gain or loss terms, the trajectory that starts from the AB may shift to a nearby periodic orbit. However, these periodic solutions are robust and remain doubly periodic with
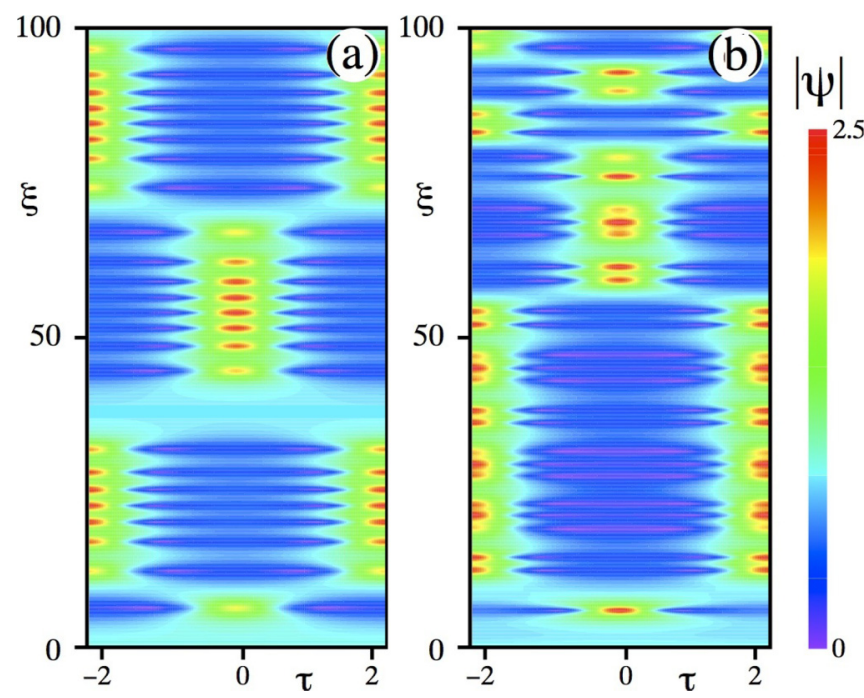

FIG. 5. False color plot of two examples of chaotic transitions between the two types of periodic solutions.
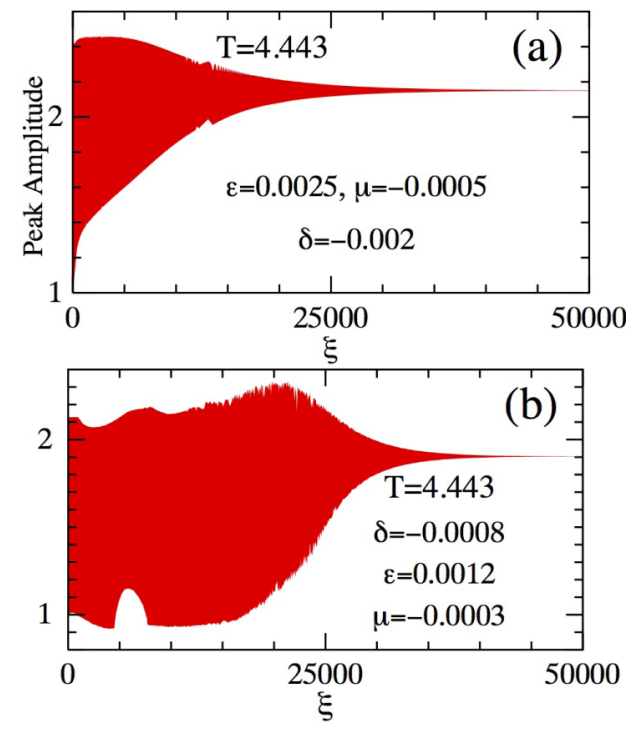

FIG. 6. Evolution of the peak amplitude (red curve) versus $\xi$ for two sets of equation parameters $\delta, \epsilon$, and $\mu$.

no prospects for converging to any stationary solution of the NLSE.

\section{SIMULATIONS WITH A PERTURBED NLSE}

As a next step, we introduced more dissipative terms to the NLSE, namely, we used the following equation:

$$
i \psi_{\xi}+\frac{1}{2} \psi_{\tau \tau}+|\psi|^{2} \psi=i \delta \psi+i \epsilon|\psi|^{2} \psi+i \mu|\psi|^{4} \psi \text {. }
$$

This equation is essentially the cubic-quintic complex Ginzburg-Landau equation [26]. It is commonly used as a master equation for passively mode-locked laser systems $[17,18]$. This equation can also provide an alternative description of soliton train generation in microcavities $[19,22]$ as the physical processes in lasers and microcavities are basically the same.

In Eq. (10), we are using the same notations as in [45]. Specifically, $\epsilon$ (being positive) represents cubic gain while $\mu$ (being negative) is the parameter responsible for the quintic saturation of the gain. The combination of three dissipative terms describes the nonlinear transmission of the cavity necessary for mode locking. The cubic gain term in (10) provides amplification while the quintic term is crucial for the stability of the solitons in the cavity [17]. All terms on the right-hand side are assumed to be small in order to maintain all solutions close to the NLSE ones.

Figure 6 illustrates two representative outcomes obtained in these simulations. Each panel in Fig. 6 shows the evolution of the peak amplitude of the field $(\max \mid(\psi(\tau, \xi) \mid, \forall \tau)$ with $\xi$. The values of the parameters $\delta, \epsilon$, and $\mu$ are given within the figures. As we can see, the parameters of the dissipative terms are small. However, they are sufficient to make a qualitative change in the wave evolution. We have chosen the same modulation frequency $(T=4.443)$ for each case which corresponds to the maximum growth rate of modulation instability. For doubly periodic solutions, the peak amplitude oscillates with small periods relative to the $\xi$ interval shown in the figures producing a curve that fills the area shown in red. Due to the differences in the parameters $\delta, \epsilon$, and $\mu$, the solutions converge to two 

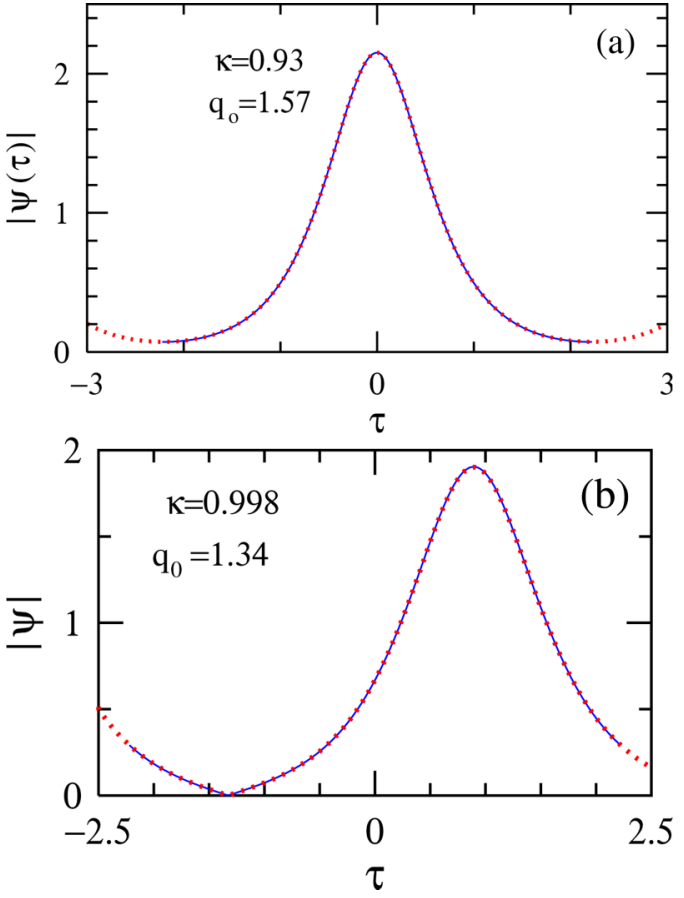

FIG. 7. Pulse profiles of two stationary solutions. The blue solid curves in (a) and (b) are the outcomes of simulations shown in Figs. 6(a) and 6(b), respectively. The red dotted curves are (a) the analytical solution (7) and (b) the analytical solution (8). The numerical and analytical curves completely coincide for the values $\kappa$ and $q_{0}$ shown in the figures.

different stationary periodic solutions. The convergence can be seen from the continuous narrowing of the red regions as $\xi$ increases. This means that the peak amplitude converges to a fixed value. This fixed value is different in the two cases shown in Fig. 6. Other simulations lead to a similar convergence to one of the two stationary solutions.

Taking small values of the dissipative parameters in Eq. (10) ensures that the solution stays close to the solution of the NLSE at each particular $\xi$. It slips from one solution to another, gradually converging to a stationary solution of the NLSE. For the two above cases, the final stages are shown in Figs. 7(a) and 7(b), respectively. In the case of simulations shown in Fig. 6(a), the convergence is straightforward without crossing the separatrix. The case shown in Fig. 6(b) is more complex and the convergence here is slower than in Fig. 6(a). After a few crossings of a separatrix, the solution converges to a moving stationary solution that can be identified as of type 2 [Eq. (8)]. Figures 7(a) and 7(b) clearly demonstrate that the convergence occurs to the solutions (7) and (8), respectively. These solutions have two free parameters $\kappa$ and $q_{0}$ that allow us to adjust the period $T$ and the amplitude of the stationary solutions to the initial period of modulation and the amplitude of the converged solutions, respectively. The numerical and analytical curves completely overlap as can be seen from Figs. 7(a) and 7(b). This shows that the converged solution in each case is indeed one of the stationary solutions (7) or (8) of the NLSE.

\section{CONCLUSION}

In conclusion, we addressed one of the major problems in physics: What is the condition for the transformation of an initially homogeneous wave state into a stable periodic pattern or train of pulses? Here, we answered this question using as paradigm the nonlinear Schrödinger equation which is one of the most widely used equations in nonlinear dynamics. We have shown that extending the original conservative system to a dissipative one described by the CGLE is essential in converging the homogeneous state into a periodic pattern.

There are many examples of this transformation in nature. A particular practical example is the generation of a periodic train of solitons out of a continuous pump in passively modelocked lasers and parametrically pumped microcavities [46]. These devices can be described using the CGLE as the master equation. Thus, our results explain the basic working principle of these devices.

\section{ACKNOWLEDGMENTS}

The authors acknowledge the support from the Volkswagen Stiftung. The work of J.M.S-C. was also supported by MINECO under Contract No. TEC2015-71127-C2-1-R, and by C.A.M. under Contract No. S2013/MIT-2790. N.D. and N.A. acknowledge support of the Australian Research Council (Discovery Projects No. DP140100265 and No. DP150102057).
[1] B. Percier, S. Manneville, and N. Taberlet, Phys. Rev. E 87, 012203 (2013).

[2] J. A. Both, D. C. Hong, and D. A. Kurtze, Physica A 301, 545 (2001).

[3] K. Mima and Y. C. Lee, Phys. Fluids 23, 105 (1980).

[4] A. M. Perego, N. Tarasov, D. V. Churkin, S. K. Turitsyn, and K. Staliunas, Proc. SPIE 9894, 98940A (2016).

[5] I. B. Burgess, W. E. Shimmell, and K. Saravanamuttu, J. Am. Chem. Soc. 129, 4738 (2007).

[6] T. B. Benjamin and J. E. Feir, J. Fluid Mech. 27, 417 (1967).

[7] V. I. Bespalov and V. I. Talanov, Zh. Eksp. Teor. Fiz., Pis'ma Red. 3, 471 (1966) [JETP Lett. 3, 307 (1966)].

[8] F. Baronio, M. Conforti, A. Degasperis, S. Lombardo, M. Onorato, and S. Wabnitz, Phys. Rev. Lett. 113, 034101 (2014).
[9] G. Millot, S. Pitois, P. T. Dinda, and M. Haelterman, Opt. Lett. 22, 1686 (1997).

[10] S. Trillo and S. Wabnitz, Opt. Lett. 16, 986 (1991).

[11] E. Fermi, J. Pasta, and S. Ulam, Los Alamos Report No. LA1940, 1955.

[12] G. Van Simaeys, P. Emplit, and M. Haelterman, Phys. Rev. Lett. 87, 033902 (2001).

[13] A. Mussot, A. Kudlinski, M. Droques, P. Szriftgiser, and N. Akhmediev, Phys. Rev. X 4, 011054 (2014).

[14] J. L. Hansen, M. van Hecke, A. Haaning, C. Ellegaard, K. H. Andersen, T. Bohr, and T. Sams, Nature (London) 410, 324 (2001).

[15] T. Aste and U. Valbusa, New J. Phys. 7, 122 (2005).

[16] T. Miura and P. K. Maini, Anat. Sci. Int. 79, 112 (2004). 
[17] A. Komarov, H. Leblond, and F. Sanchez, Phys. Rev. E 72, 025604(R) (2005).

[18] A. Zavyalov, R. Iliew, O. Egorov, and F. Lederer, J. Opt. Soc. Am. B 27, 2313 (2010).

[19] T. J. Kippenberg, R. L. Holzwarth, and S. A. Diddams, Science 332, 555 (2011).

[20] J. Kim, F. X. Kartner, and M. H. Perrott, Opt. Lett. 29, 2076 (2004).

[21] S. B. Papp, K. Beha, P. Del'Haye, F. Quinlan, H. Lee, K. J. Vahala, and S. A. Diddams, Optica 1, 10 (2014).

[22] S. Coen, H. G. Randle, T. Sylvestre, and M. Erkintalo, Opt. Lett. 38, 37 (2013).

[23] S. T. Cundiff and J. Ye, Rev. Mod. Phys. 75, 325 (2003).

[24] N. R. Newbury, Nat. Photonics 5, 186 (2011).

[25] S. A. Diddams, J. C. Bergquist, S. R. Jefferts, and C. W. Oates, Science 306, 1318 (2004).

[26] I. S. Aranson and L. Kramer, Rev. Mod. Phys. 74, 99 (2002).

[27] H. C. Yuen and B. M. Lake, Phys. Fluids 18, 956 (1975).

[28] L. F. Mollenauer, R. H. Stolen, and J. P. Gordon, Phys. Rev. Lett. 45, 1095 (1980).

[29] C. R. Menyuk, J. Opt. Soc. Am. B 10, 1585 (1993).

[30] N. J. Zabusky and M. D. Kruskal, Phys. Rev. Lett. 15, 240 (1965).

[31] M. Remoissenet, Waves Called Solitons: Concepts and Experiments (Springer, Berlin, 2003).
[32] W. H. Renninger and F. W. Wise, Nat. Commun. 4, 1719 (2013).

[33] A. C. Scott, F. Y. F. Chu, and D. W. McLaughlin, Proc. IEEE 61, 1443 (1973).

[34] S. K. Turitsyn, B. G. Bale, and M. P. Fedoruk, Phys. Rep. 521, 135 (2012).

[35] A. Blanco-Redondo et al., Nat. Commun. 7, 10427 (2016).

[36] N. N. Akhmediev, V. M. Eleonskii, and N. E. Kulagin, Theor. Math. Phys. 72, 809 (1987).

[37] Handbook of Mathematical Functions, edited by M. Abramowitz and I. A. Stegun (NBS, New York, 1964).

[38] N. Akhmediev, V. M. Eleonskii, and N. Kulagin, J. Exp. Theor. Phys. 62, 894 (1985).

[39] N. Akhmediev and V. I. Korneev, Theor. Math. Phys. 69, 1089 (1986).

[40] B. Frisquet, B. Kibler, and G. Millot, Phys. Rev. X 3, 041032 (2013).

[41] C. Mahnke and F. Mitschke, Phys. Rev. A 85, 033808 (2012).

[42] J. P. Boyd, SIAM J. Appl. Math. 44, 952 (1984).

[43] A. Calini and C. M. Schober, Nat. Hazards Earth Syst. Sci. 14, 1431 (2014).

[44] O. Kimmoun et al., Sci. Rep. 6, 28516 (2016).

[45] J. M. Soto-Crespo, N. Akhmediev, and G. Town, J. Opt. Soc. Am. B 19, 234 (2002).

[46] Z. Liu, S. Coulibaly, M. Taki, and N. Akhmediev, Opt. Lett. 42, 2126 (2017). 